\title{
THE PRODUCTION OF $\mathrm{CO}_{2}$ BY THE TYPHOII) \\ BACILLUS AND THE MECHANISM OF THE RUSSELL DOUBLE SUGAR TUBE
}

\author{
HENRY J, NICH OLS
}

From the Dizisian of Laborataries of the Army Medical School, Washington, D. C.

In the course of some work on the nature of the changes in the Russell double sugar medium during the growth of the typhoid bacillus, a fact of considerable theoretical interest was observed, namely, that the typhoid bacillus produces $\mathrm{CO}_{2}$ in appreciable amounts. This fact has apparently escaped previous observation, but seems definite in spite of the absence of any visible evidence of gas.

The explanation of the appearance of the double sugar tube as given in the original description and repeated elsewhere, is as follows : ${ }^{1}$

"The entire point of the medium rests upon the difference in changes produced by the growth of the typhoid bacillus under aerobic and under the imperfect anaerobic conditions found in the butt of the tube, where the bacillus obtains its oxygen by breaking down the glucose with the liberation of considerable acid; on the surface, however, in the presence of free oxygen, no acid is formed."

In this explanation no place is found for the fact that the typhoid bacillus ferments glucose with acid end-products under both aerobic and anaerobic conditions. It occurred to the writer that the glucose might be fermented in the slant as well as in the butt and that the small amount of acid formed from the $0.1 \%$ glucose might volatilize and leave the medium apparently unaffected. In order to test this hypothesis several experiments were made with results which support this explanation as one important factor in the mechanism of the tube.

When a double sugar tube inoculated with the typhoid bacillus (Rawlings), is coupled up ${ }^{2}$ in an anaerobic system of pyrogallic acid and $\mathrm{KOH}$, the appearance of the slant is the same as usual. In other words, in an atmosphere free of oxygen, no acid is apparently produced, as should be the case according to the original explanation. If a volatile acid is assumed, however, the neutral slant can be readily explained by absorption of the acid by the $\mathrm{KOH}$.

Received for publication Marçh 17, 1921.

1 Russell, F. F.: Jour. Med. Research, 1911, 25, p. 227.

2 Nichols, H. J., and Scbmitter, F.: Ibid., 1906, 15, p. 113. 
In an inoculated double sugar tube covered with oil, the slant becomes strongly acid. This result, in the light of our knowledge of oil seals, ${ }^{3}$ can be explained by the retention of acid better than by the exclusion of oxygen.

In an inoculated double sugar tube sealed by heat or paraffin the slant becomes acid. This result also can be explained better by assuming a retention of a volatile acid rather than an exhaustion of oxygen.

When an inoculated double sugar tube is coupled up in a tight system with an uninoculated tube, the slant of the second tube becomes acid as well as the first; in other words, some volatile acid passes over to the uninoculated tube.

Finally a set of glucose agar Andrade tubes of various strengths from $0.01 \%$ to $1 \%$ by tenths, inoculated with the typhoid bacillus, gives these results :

TABLE 1

Restlts of Inoculation of Glucose Andrade Tubes with the Typhoid Bachlins

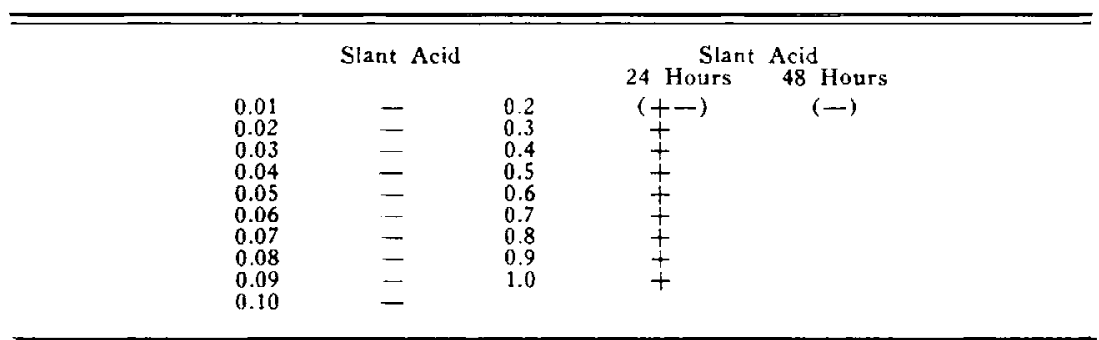

These results indicate that the typhoid bacillus ferments glucose in the slant under aerobic conditions. The acid products of fermentation are evident above $0.3 \%$ and were undoubtedly present in small amounts in the lower percentages, but leave no evidence of fermentation on account of their escape and neutralization. This latter additional factor is indicated by the appearance of the $0.2 \%$ glucose tule which shows some acid on the slant in 24 hours, but none in 48 hours. It is common knowledge among users of the double sugar tube that a distinct alkaline reversion occurs after several days. It has recently been shown by Ayers and Ruff ${ }^{4}$ that simultaneous acid and alkaline fermentations occur with members of the colon-aerogenes group.

It is seen that the volatile acid, of which evidence has been presented, acts very much like $\mathrm{CO}_{2}$. In fact, the appearance of a litmus double

3 Gates, F. L., and Olitsky, P. H.: Jour. Exper. Med., 1921, 33, p. 51.

- Jour. Infect. Dis., 1918, 23, p. 188. 
sugar tube, under the varying conditions given above, can be exactly duplicated by saturating sterile medium with $\mathrm{CO}_{2}$. But the typhoid bacillus does not produce $\mathrm{CO}_{2}$ according to any authority. The most complete work on the acid products of the growth of the typhoid bacillus in glucose is that of Harden ${ }^{5}$ who found about $40 \%$ of lactic acid, $17 \%$ of acetic acid, $17.5 \%$ of formic acid, a trace of succinic acid and $10 \%$ alcohol. "He points out that with forms like B. typhosus, formic acid must be produced from the fractions of the molecule which with $\mathrm{B}$. coli yield $\mathrm{CO}_{2}$ and $\mathrm{H}_{2} .{ }^{\prime}$ As acetic and formic acids are volatile, it was at first thought that they might be responsible for the appearance of the tube. About this time, however, the writer became acquainted with the work of Ayers, now in press, on the production of $\mathrm{CO}_{2}$ by streptococci. Ayers used the little known but practical Eldredge tube, ${ }^{7}$ and showed that $\mathrm{CO}_{2}$ is produced not only from sugars but also from proteins.

TABLE 2

Results of Experiments With Eldredge Tube

\begin{tabular}{|c|}
\hline 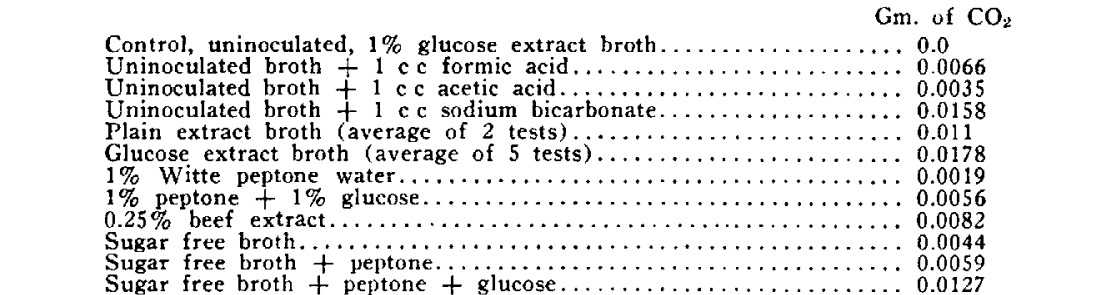 \\
\hline
\end{tabular}

THE PRODUCTION OF $\mathrm{CO}_{2}$ BY THE TYPHOID BACILLLS

Some experiments with the Eldredge tube yielded the following significant results. (In all the experiments $50 \mathrm{c} \mathrm{c}$ of medium and the Rawlings typhoid strain were used, $25 \mathrm{c}$ c of $0.1 \mathrm{~N} \mathrm{Ba}(\mathrm{OH})_{2}$ were placed in the absorbing tube. After 24 hours' growth, this was titrated with $0.1 \mathrm{~N} . \mathrm{HCl}$ to a loss of color with phenolphthalein and then to a pink color with methyl orange. The difference between 25 and the first figure [for example 17.4], such as 7.6 gives a result in terms of $0.1 \mathrm{~N}$, $\mathrm{H}_{2} \mathrm{CO}_{3}$. This figure was in turn multiplied by 0.0022 to give gm., of $\mathrm{CO}_{2}$ ).

This table shows that $\mathrm{CO}_{2}$ is produced by the typhoid bacillus both from glucose and from proteins. It also shows that the decomposition of formic acid is not the source of much of the $\mathrm{CO}_{2}$. The relative

s Jour. Chem. Soc:, 1901. 79. n. 610

- Winsiow, Kligler and Rothberg: Jaur. Bacteriol., 1919, 4, p. 429.

7 Eldredge, E. E., and Rogers, L. A.: Centralhl. f. Bakteriol., 1914, 40, p. 5. 
amount of $\mathrm{CO}_{2}$ to other acids is of importance and incidentally has a direct bearing on the explanation of the double sugar tube. In terms of $0.1 \mathrm{~N}$ acid and alkali, the ratio was found to be 7.6 to 9.5 or about $45 \%$ of the total acid produced is $\mathrm{CO}_{2}$.

$\mathrm{CO}_{2}$ is soluble in about $60 \%$ its own volume in water at $760 \mathrm{~mm}$. of $\mathrm{Hg}$ and $35 \mathrm{C}$. and the fallacy of the ordinary $\mathrm{CO}_{2}$ determination has been pointed out by Keyes ${ }^{8}$ and Clark. ${ }^{9}$ It is evidently necessary to use more exact methods such as those of Harden, Keyes, Rogers, Clark and Davis, ${ }^{10}$ and Fildredge and Rogers. Just why the $\mathrm{CO}_{2}$ was missed in Harden's work on the typhoid bacillus, and by other observers, the writer is unable to say. Nor can he discuss the physical chemistry of $\mathrm{CO}_{2}$. But if the Eldredge is reliable, as it seems to be, there is no doubt about the production of $\mathrm{CO}_{2}$ in important amounts. Several other organisms, such as paratyphoid A and B, the dysentery bacilli and B. subtilis, affect the double sugar mediums in somewhat the same way as the typhoid bacillus. All these organisms yield consiclerable amounts of $\mathrm{CO}_{2}$ in the Fildredge tube from dextrose brotl.

The explanation of the double sugar tube is largely an academic question and does not affect the value of this medium, which with slight variations in indicators or sugar content has come into routine use in the diagnosis of organisms of this group. But, of course, the most truthful explanation will link up best with other observations and will be least confusing to students.

\section{SUMMARY}

$\mathrm{CO}_{2}$ is produced by the typhoid bacillus in significant amount: both from sugars and from proteins.

The appearance of the Russell double sugar tube during the growth of the typhoid bacillus is not due to direct oxygen requirements. It is due (a) to the retention of $\mathrm{CO}_{2}$, in the butt of the tube and its escape from the slant and (b) to alkaline reversion of other acids.

3 Jour. Med. Res., 1909, 21, 13. 69.

- Science, 1913, 38, p. 669.

10 Jour. Infect. Dis., 19:4, 14, p. 411. 